\title{
3D Revision Control Framework
}

\author{
Jozef Doboš, Anthony Steed \\ University College London
}

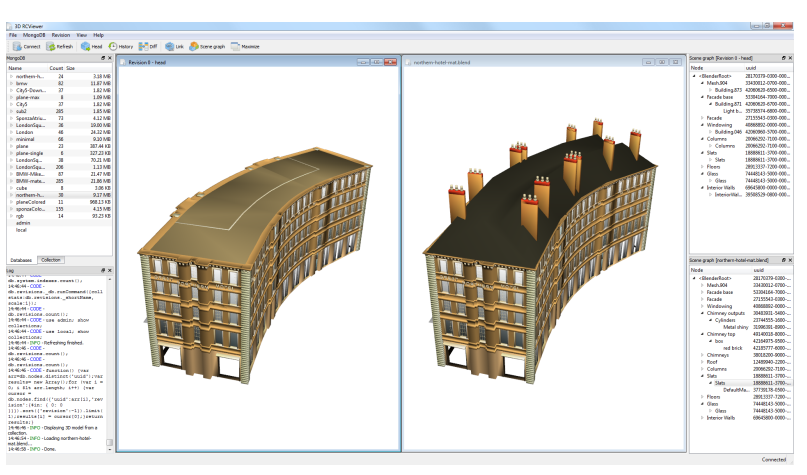

(a) 3D RCViewer

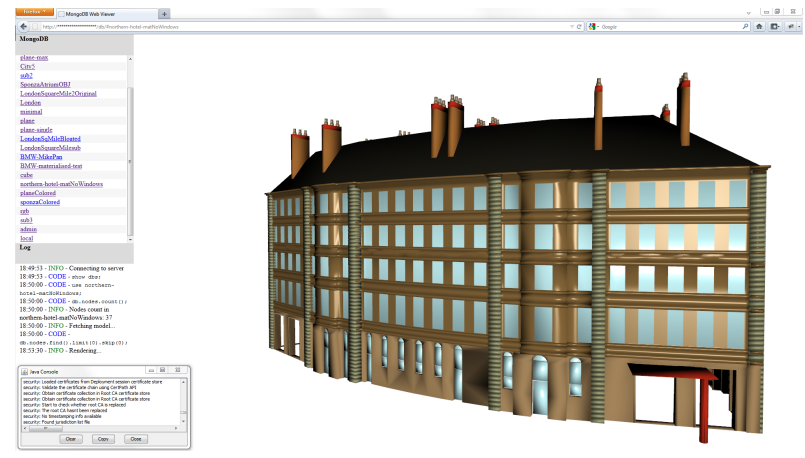

(b) 3D Web Client

Figure 1: We define a novel 3D Repository that supports version tracking and asset distribution built on top of a NoSQL database. In (a), the $3 D$ Revision Control Viewer enables repository inspection, individual revisions retrieval as well as conflict resolution and merging. In addition, the 3D Web Client, (b), remotely visualizes the contents of the repository in a web browser. Model courtesy of Network Rail.

\begin{abstract}
The maintenance of assets in a large 3D scene can involve many authors with potentially different skills and different modeling tools. The standard paradigm of collaborative editing is sharing of files between various instances of applications. This presents problems, not limited to maintaining consistency of the models and dealing with concurrent edits in the same part of a scene. In this paper, we present a novel framework for non-linear revision control and online distribution of 3D assets. This framework supports concurrent asynchronous 3D editing of models and tracking of multiple revisions so that they can be integrated at a later date. It thus provides similar functionality to file-based revision control systems, but is built around a NoSQL database, hence avoids the constraints of file based storage. The framework supports distributed editing over the Internet and additional lightweight clients in web-browsers with WebGL support.
\end{abstract}

CR Categories: I.3.4 [Computer Graphics]: Graphics UtilitiesSoftware support; I.3.4 [Computer Graphics]: Graphics UtilitiesGraphics editors

Keywords: 3D database, version tracking, revision history, nosql, webgl client, java liveconnect

\section{Introduction}

With the recent progress in the capabilities of the graphics processing units, there is a desire for even larger and more detailed mod-

\footnotetext{
*e-mail: j.dobos@cs.ucl.ac.uk
}

els. Whilst massive procedurally generated datasets have long been used in engineering and scientific visualizations, in domains ranging from cultural heritage [Koller et al. 2010] through to computer games there is a growing need to maintain extensive 3D scenes that might be edited by multiple users concurrently.

The main motivation for this work are the drawbacks of storing 3D models as files on a file system. Each user loads a particular scene into a modeling tool, modifies it and then re-saves the whole file again, making the unit of access to the scene the file itself. If multiple users want to edit the same part of a scene, they need to access the same file but without extra infrastructure, managing this access and resolving conflicts becomes infeasible. The machine memory is a strict limit too as large scenes exceed hardware capabilities by many times. On the other hand, deciding how a large scene should be partitioned into multiple files is a non-trivial task either. Splitting objects or meshes across several files raises an important question: How to manage edits that involve multiple objects and how to keep their different versions in sync? Revision control and asset management systems are limited, yet, common solutions to this problem, see $\S 2.2$. Hence, there is a need for a centralized yet flexible framework that would enable transparent asset management and would support multiple users with distributed access. To be widely adopted, it has to provide efficient storage, revision control and interface with the existing editing packages while not enforcing any particular modeling paradigms on its users.

Integrated revision control and editing for images has recently been studied by Chen et al. [2011]. In their versioning system for 2D images, they created a plug-in to an image editor that tracks user actions to form revisions. Our work targets a different type of assets, 3D models. What is more, it is our belief that we should not, initially, rely on any specific modeling tool, but rather deal with $3 \mathrm{D}$ files external to the editor. In search of a suitable solution, we exploit the recent developments in the NoSQL database (DB) technology and build a non-linear revision control framework for 3D assets upon it. NoSQL databases avoid rigid table structures and tend to be optimized for large read-write operations. It is thus possible to store models in a DB and, due to its flexibility, also track other associated data such as semantic relationships, individ- 
ual revisions and even shaders. Once in a DB, access is implicitly supported in a distributed manner via a dedicated query language. The prototype implementation that we present uses the open source MongoDB [Membrey et al. 2010] as its 3D repository. Our primary DB front-end supports repository visualizations, revision control including branching and merging via a novel 3D Diff tool, firstly introduced in [Doboš and Steed 2012]. A secondary DB front-end further renders selected revisions inside the common web browsers using a straightforward combination of Java applet, JavaScript and WebGL.

The main contributions of this paper are the unification of design and revision control of 3D assets. Hence we show:

- How a scene graph representation of 3D assets can be stored inside a NoSQL database (MongoDB).

- How the same DB can be extended to store revision history.

- A stand-alone interface for revision control management which loads models into the DB and supports repository previewing and conflict resolution.

- A secondary read-only interface that renders models from the repository inside the common web browsers.

We demonstrate the feasibility of our framework on examples with up to several million polygons. We feel confident in arguing that our approach would be suitable for large-scale collaborative 3D editing. Given the type of DB we employ is proven to scale massively, the potential long-term benefits are extensibility for fitting into existing modeling pipelines and even open crowd-sourcing of 3D models.

\section{Related Work}

An important debate which we do not have the space to do justice here, revolves around the relative merits of using file systems over DBs for large data storage. In general, file systems provide mechanisms to store, localize and retrieve files on local or remote devices. There are, however, several issues with relying on file systems to support large-scale modeling. Whilst revision control systems are popular, they aggregate edits at a file level. If a model is stored in plain text, it may be that edits can be tracked on a per-line basis. Even though formats such as COLLADA [Barnes and Finch 2008] are designed to facilitate the digital asset interchange, the scale and type of edits often means that the ASCII changes are pervasive across the entire file. Many 3D formats are binary so, in the worst case, a whole file needs to be tracked. If a scene is split across several files, a description of how these fit together must also be stored. Commonly, this is separated from individual models, though, some formats such as X3D [Web3D Consortium 2008] can recursively include files, thus serving both purposes. Loading of multiple files into editors is usually not supported and if it is, it requires careful management (e.g. XRefs in Autodesk 3ds Max).

\subsection{Databases}

There are several advantages of using a DB. First is the central point of control. Even though a DB might support sharding across multiple servers, logically, there is only a single point of access. Second is the usability locally or over the network. Third is the unit of access which is defined by the schema designer. 3D data can therefore be stored as binary per-mesh documents or even as per-vertex entries. Final is the support for some or all of the ACID data integrity principles [Connolly and Begg 2004].

Spatial databases [Rigaux et al. 2001] originate from the geographic information systems domain. They store features such as points, lines, areas and their associated features like heights, names, etc. A few commercial and open source databases support spatial data directly, in particular Oracle Spatial [V. Kothuri et al. 2012] and PostGIS for PostgreSQL [Obe and Hsu 2011]. In comparison, our framework focuses on distributed access to and editing of 3D assets. Spatial DBs focus on 2D geometry and metadata, not 3D models and other visual properties. They also do not support the type of revision histories that we require.

\subsection{Asset Management and Version Control}

The management of graphics assets is an important facility in many industries. Whilst some guidelines and descriptions of best practices exist, [Jacobsen et al. 2005] or [Austerberry 2006] for example, there are few standards. Generally, the process can be split into two tasks: storage on a file system and history tracking of file names and associated metadata. Such tracking usually involves strict naming conventions or fixed file system hierarchy. Optionally, to reduce the storage requirements, revision control can be employed. In some cases, tracking might use a DB to store file histories as done in some games companies. If, for example, Perforce [Wingerd 2005] is used to store files and Oracle DB to track the history, creating game levels involves querying the DB to get a set of assets, copying them locally and processing them before loading onto the target console or a PC. Dedicated tools, such as Temerity Pipeline or Alienbrain provide digital asset management and other facilities [Jacobsen et al. 2005]. High-end CAD provides similar functionality, such as Dassault Systemes' Enovia [Dassault Systemes 2011] or Bentley's AssetWise [Bentley Systems 2011]. Unity3D, for instance, offers Asset Server, a networked service for asset management that can utilize various revision control systems [Unity Technologies 2010]. However, those systems that are commonly used, e.g. Subversion [Pilato et al. 2008] or Perforce, are better at managing text than binary files. Difference tools (simple diff) make these good at managing and integrating source code but not binary data [Hunt et al. 1998].

Edit Logging A problem related to asset control is how users manage different versions of a file to perform collaborative editing [Dourish 1995]. One approach to merging changes on binary data is to track editing operations the users make, expecting that this log can inform the process [Wäsch and Klas 1996]. Such a concept has been realized by [Chen et al. 2011] for 2D images. VisTrails Provenance Explorer for Maya [VisTrails, Inc. 2012] provides the state-of-the-art side-by-side differencing of 3D revisions. Despite capturing the edit history in a MySQL database, the models are stored locally. Similarly, Denning et al. [2011] log edits in Blender. By label clustering via several layers of regular expressions, they create an interactive playback of modeling history. This, however, captures only single-user mesh edits. In order to support a wide variety of editing tools, we have decided not to rely on edit logging.

\subsection{Network Distribution}

The support of graphics distribution over the network is in itself a large research area [Steed and Oliveira 2009]. Systems such as Distributed Open Inventor [Hesina et al. 1999] supported the sharing of full scene graphs and subsequent real-time edits. Similar live editing and viewing based on a custom transfer protocol was implemented in the Uni-Verse platform [Brink and Steenberg 2011]. In contrast, a scene graph rendering engine SceneJS [Kay et al. 2009], provides a JavaScript API for WebGL. Its JSON-based representation can easily be transported over the network and stored in a DB. Built around a very similar technology is a recent MIT spinoff, Sunglass [DeBiswas and Rao 2012], which offers a proprietary WebGL platform for sharing of 3D models. These are uploaded through a web browser into a centralized server and further pushed to addi- 


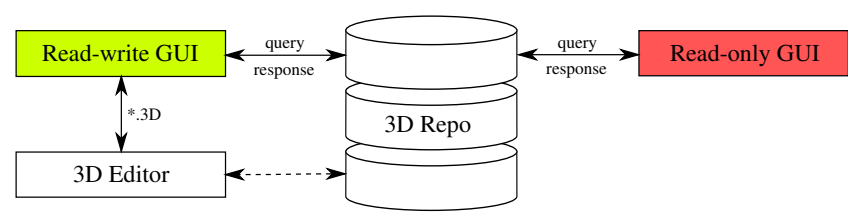

Figure 2: Conceptual framework overview. Editing software exports $3 D$ file into a tier 1 (read-write) GUI which facilitates version control by storing the decomposed scene inside a $3 D$ Repository. Tier 2 (read-only) GUI, a secondary DB client, further visualizes the contents of the repository without write privileges. Dashed arrow shows the possible future connection, see $\S 7$.

tional clients. Unlike our framework, it does not provide version control nor open access to its models. Other solutions concentrated on directly integrating models into the Document Object Model (DOM) structure itself. XML3D by Sons et al. [2010], for example, embeds 3D data represented as XML inside the HTML. This relies on the parsing capabilities of a web browser which might be a limiting factor for large 3D scenes. Such problems are solved by X3DOM [Behr et al. 2010] which integrates X3D directly into the DOM. By encoding large geometry as images it circumvents the parsing limitations. Just like other techniques, these methods suffer from a text-based representation when tracking revision histories.

\subsection{Summary}

While the existing management systems are promising, they are either not open, difficult to implement or deal with scenes at an inflexible per-file level. Our aim is to create one DB to store the whole scene and its revision history. This can potentially save a significant amount of storage over equivalent systems using filebased versioning. It is also extensible to include all types of assets such as materials, textures, shaders etc. and provide new means of transmitting 3D data across the Internet.

\section{Framework Overview}

Nowadays, much of the $3 \mathrm{D}$ content is procedurally generated or manipulated by custom tools. Collaborative editing of scenes produced by even a standard set of modeling packages is not as straightforward as might be required by the industry. In order to separate the modeling from its long-term storage, it is necessary to become agnostic to any specific editing software. We, therefore, propose to load and save various 3D file formats via our framework that stores a unified scene graph representation and its associated delta changes in a centralized repository.

Our revision control framework, outlined in Fig. 2, is built around a domain specific database, a.k.a. $3 D$ repository. The role of the repository is to store $3 \mathrm{D}$ assets and to provide interfaces and conventions to add, modify and extract delta changes on them. By supporting a standard web-accessible front-end, it enables connections to modeling packages via intermediary tools, or simply to a viewer that visualizes selected revision from the DB. Conflict resolution is achieved via a visual $3 D$ Diff which highlights incompatible edits and offers quick merge suggestions. Furthermore, the framework supports retrieval of any full or partial revision that can be exported as a new 3D file. We distinguish two tiers of repository front-ends, those with a read-write and those with a read-only access, see $\S 5$.

The main features of our framework include:

1. Storage of a wide range of assets such as geometry, materials and shaders. In principle, the repository is extensible to a variety of format and domain specific data structures.

2. The ability to preserve delta changes on $3 \mathrm{D}$ assets alongside their non-linear revision history within a standard DB (acting as our 3D repository).

3. Easy access control and full querying potential of DBs including sub-object retrieval.

4. An interactive user-driven approach to mesh differencing and conflict resolution (3D Diff).

5. A web browser interface for repository inspection.

6. Its extensibility potential for integration into existing modeling pipelines of creative industries and automation of tasks (e.g. nightly backups, hourly conflict resolution, etc.).

\subsection{D Repository}

Spatial databases such as Oracle Spatial or PostGIS, see $\S 2.1$, offer flexibility for generic spatial queries, however, they consume significant amount of storage space reducing the performance of the underlying DB. 3D files, on the other hand, group every scene object into a binary or plain-text representation that does not support sub-object queries and requires a whole file to be loaded into the modeling software for editing.

To our advantage, large models are usually composed of several separate components such as meshes, materials and the like, interlinked via their semantic relationships. The building model in Fig. 1, for example, consists of separate meshes for the roof, façade, etc. We exploit this natural partitioning of 3D assets and base our versioning framework upon it. To be able to capture a 3D scene alongside its revision history in a single DB, we break this problem into two conceptually different, nevertheless identical in nature, data structures: scene graph $(S G)$ and revision history $(R H)$.

\subsubsection{Scene Graph}

Previously [Zeleznik et al. 2000] and more recently [Berthelot et al. 2011] used a scene graph as a data structure to intermediate between various 3D applications. Similarly, we rely on a scene graph as a file-format-independent representation that can easily be manipulated and stored in a DB. Unfortunately, no commonly accepted structure exists and different implementations impose their own restrictions on the scene hierarchy. We, therefore, rely on a generic definition of a scene graph.

Without loss of generality, we treat $S G$ as a directed acyclic graph $(D A G)$ with a single root node $n_{\text {root }}$, where each node $n \in S G-\left\{n_{\text {root }}\right\}$ is stored in its local coordinates with an associated transformation $T_{n}$. When recursively applied from the root to each child node, these transformations together define the global position in world coordinates. This supports instancing suitable for real-time rendering but also the automatic merge functionality as discussed in $\S 4$. In this context, a $S G$ node represents any scene specific data such as meshes, transformations, cameras, textures as well as animations, bones and shaders. Each such node is recorded as a binary "document" with preserved relational information with regards to the other nodes. Therefore, it is possible to retrieve any sub-part of the entire graph if required. In contrast to a single 3D file, such polymorphic representation offers the desired flexibility yet establishes a suitable compromise between storage efficiency and querying potential of spatial databases. This design supports all the common constituents of 3D assets. However, we do acknowledge that some of these (e.g. textures) can be extremely large. Storing them would be non-trivial, hence, we leave it open for future exploration. For instance, our prototype implementation is currently limited to $16 \mathrm{MB}$ per-document due to the type of $\mathrm{DB}$ in use, see $\S 7.1$. Nevertheless, many 3D file formats impose sim- 
ilar restrictions (e.g. X3D with a maximum of $2^{16}$ vertices). Furthermore, it shall be noted that even though a single vertex change would require an entire new mesh document to be stored in the next revision, we consider this a delta change. This is because only a partial modification of the $S G$ is preserved, not the whole scene.

\subsubsection{Revision History}

As defined in Git and many other versioning systems [Sink 2011], a revision history that supports non-linear branching and, more importantly, merging can be modeled as a DAG. Therefore, if a scene graph can be stored in a DB, a revision history can be stored, too. Here, each $R H$ node represents a new revision with a corresponding incremental non-negative revision number (R\#) and a set of associated delta changes on the scene graph. To match previous versions of any $S G$ node to its new revision, all components of a 3D asset are first assigned a unique identifier (ID) that is shared among the same nodes (and editing software) over time. Each $S G$ node has to further carry the revision number it belongs to. We, therefore, combine these into a tuple

$$
\text { revision metadata }=(\mathrm{ID}, \mathrm{R \#}) .
$$

In relational databases terms, such a tuple would constitute a composite primary key on the $S G$ table, so that every node can contribute exactly once to exactly one revision. The possibility of losing such metadata during the round-trip from the editing software to our revision framework is addressed in $\S 7.1$.

\subsection{Repository Front-ends}

In order to support revision management as defined by our framework, we rely on two tiers of repository front-ends to assume the responsibility of version tracking and model retrieval, see Fig. 2.

Tier 1 In our framework, 3D files are used as a temporary representation for the purposes of transportation in between editing systems and the long-term storage. Repository front-ends with read-write access have to, therefore, enable importing and exporting of various 3D file formats. Because the framework does not support asset locking yet, a 3D differencing UI, as outlined in $\S 4.1$, has to be built-in. $\S 5.2$ provides an example.

Tier 2 On the other hand, read-only applications have to support only a subset of the functionality, most importantly revision retrieval and visual inspection of the repository. A variety of lightweight applications can be built in this way. In $\S 5.3$, we present a client-side viewer that renders the contents of the repository inside WebGL enabled web browsers.

In contrast, it is possible to leverage the plug-in frameworks of popular modeling packages and connect them to the DB directly, see dashed arrow in Fig. 2. We, however, believe that our solution should not rely on any specific editing tool, but rather support a variety of them.

\section{Revision Management}

To be able to support a non-linear revision control similar to a filebased version tracking, we define the trunk to represent the main development stream while potentially multiple branches stand for additional side streams that can eventually be merged back to the trunk. Several features are considered to support full revision control. Firstly, there is the need to retrieve the latest (head) revision as well as to identify any previous version in order of creation so that any one of them can be selected. With large scenes in mind, we support sub-graph retrieval where only some parts of the original 3D model are returned. Next, the framework allows for nodes to be deleted so that they do not contribute to the scene graph from the next revision onwards. Without proper locking ${ }^{1}$, potential conflicts on $S G$ modifications as well as during merging can occur. These are efficiently resolved by our novel 3D Diff interface which highlights conflicting scene objects and offers fast conflict resolution without the need for a $3^{\text {rd }}$-party editor. However, the framework can easily be extended to enforce access control, see $\S 7.3$.

Revision Retrieval In order to query any version of a scene from the repository, a revision has to be selected. This together with all of its ancestral $R H$ nodes defines a search space in which individual $S G$ nodes can be found. Therefore, to retrieve the entire $3 \mathrm{D}$ scene that corresponds to a selected revision, only the latest versions (highest revision numbers) of all the $S G$ nodes from this search space are to be queried.

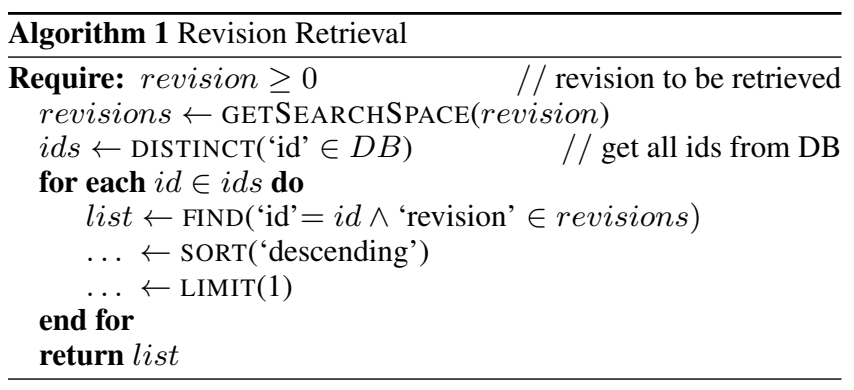

Assuming the knowledge of this search space, i.e. of all possible revision paths that lead to a selected revision, labelled revisions, Algorithm 1 returns a list of the latest $S G$ nodes that belong to a requested revision. In order to retrieve only a sub-section of the entire scene graph, it is possible to specify individual ID values to be returned, replacing the distinct('id') call in Algorithm 1.

Revision Commit Initially, when the repository is empty, the user commits an entire 3D scene as revision 0. Further commits replace individual $S G$ nodes corresponding to delta changes, defining a new head revision. However, trying to commit changes on a node which is at least one revision behind the head, hence out of sync, results in a conflict. In such a case, the user has to merge the local version (or branch) to the repository.

Node Deletion In order to support previous revisions, when deleting a $S G$ node, it is only marked as "deleted". Hence, if a node is to be removed from the repository, a NULL type document with the same ID is inserted in the next revision. In addition, the entire sub-graph of this node has to be recursively checked. If any of the descendant nodes has no other parents, it also has to be deleted.

\subsection{D Diff and Merge}

Given the assumption from $\S 3.1 .1$ that $S G$ nodes are stored in local coordinates with associated path that leads to a global transformation, even changes that seemingly affect the same piece of geometry can automatically be merged. Suppose that User $_{1}$ in Fig. 1 checked out the building model and increased its height. By doing so, the roof mesh was displaced but not modified. Further suppose that User $_{2}$ added chimneys in the meantime but was not aware of the height adjustment. Due to mesh modifications being independent of its position, User 2 can commit using the auto merge functionality without the need to resolve any conflicts.

\footnotetext{
${ }^{1}$ Locking is not supported in standard revision repositories (e.g. Git) either as it is a file-system issue.
} 


\begin{tabular}{cc|c}
\multicolumn{3}{c}{ 2-way } \\
A & B & Result \\
\hline$\bigcirc$ & $\bigcirc$ & $\bigcirc$ \\
\hline$\oplus$ & $\otimes$ & conflict
\end{tabular}

\begin{tabular}{c|cc|c}
\multicolumn{5}{|c}{ 3-way } \\
Ancestor AB & A & B & Result \\
\hline$\bigcirc$ & $\bigcirc$ & $\bigcirc$ & $\bigcirc$ \\
$\bigcirc$ & $\oplus$ & $\oplus$ & $\oplus$ \\
\hline$\bigcirc$ & $\oplus$ & $\bigcirc$ & $\oplus$ \\
$\bigcirc$ & $\bigcirc$ & $\oplus$ & $\oplus$ \\
$\bigcirc$ & $\oplus$ & $\otimes$ & conflict
\end{tabular}

Table 1: A schematic representation of a 2-way vs. a 3-way diff with suggested merge results. Each scene graph node can be modified in the A or B revisions of the same model.

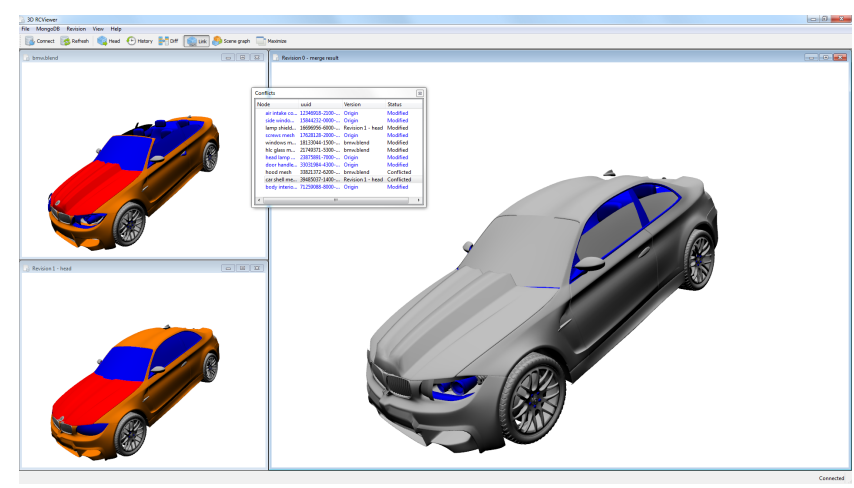

Figure 3: 3-way 3D diff. Two versions of the same heavily instanced model with nearly $200 \mathrm{k}$ polygons are loaded and merged into their common ancestor (right). Conflicting changes are in red, non-conflicting in blue and the current selection in orange.

Concurrent edits on the same $S G$ node such as modifications of the individual vertices or even texture coordinates by multiple artists are considered conflicting. These cannot be automatically merged and would need to be exported into a 3D file and resolved manually in a dedicated vertex-level editor. Modeling packages such as Autodesk 3ds Max, Maya (http://autodesk.com) or Blender (http://blender.org) superimpose the meshes but do not aid the merging process any further. However, most of the time it would be satisfactory to preserve only one of the conflicted $S G$ nodes, discarding the other version entirely.

We, therefore, implement a $3 D$ Diff tool firstly introduced in [Doboš and Steed 2012]. Unlike previous works on shape variations that need to establish the model correspondence (such as [Jain et al. 2012]), we match $S G$ nodes based on their assigned ID. Any discrepancies (marked as $\oplus, \otimes$ in Table 1) in two corresponding $S G$ nodes are treated as conflicting edits and are highlighted accordingly (see Fig. 3). The user has to indicate which of the two versions to keep in the merged result. If we further integrate the knowledge about a common ancestor of the two models, a.k.a. 3way diff [Mens 2002], as shown in Table 1, we can automatically resolve additional conflicts. If one of the conflicted $S G$ nodes is the same as in the ancestral model, the other must be the intended modification. Nevertheless, we consider this only an automated suggestion that requires user's approval as such changes could still interfere with the remaining parts of the model.

\section{Prototype Implementation}

We demonstrate the feasibility of our framework by implementing a 3D Repository within a standard release of MongoDB. Two distinct DB front-ends, one per tier ( 33.2$)$, offer revision control management and repository visualization as defined in Fig. 2. Tier 1 application, the 3D Revision Control Viewer (3D RCViewer), is a stand-alone GUI that supports all of the revision management functionality outlined in $\S 4$. On the other hand, the 3D Web Client (3D WC) offers read-only repository access that renders its contents within WebGL enabled web browsers.

\subsection{MongoDB}

Dedicated graph-based databases such as Pregel [Malewicz et al. 2009] offer large-scale distributed processing capabilities. We, however, do not require complicated graph traversal computations but rather an efficient and more importantly a unified way of encoding various object-like data. In contrast to traditional relational DB systems, NoSQL DBs store polymorphic collections of structured data that offer great flexibility and ease of access. We make use of the MongoDB which is an open source C++ NoSQL database that utilizes Binary JSON (BSON) [2010] documents for storage, querying and data transmission. This little-endian serialized representation employs $\mathrm{C}$ data types (byte, int32/64, double) that can easily be decoded making the DB highly responsive. In contrast to JSON, BSON offers several additional binary entries that are suitable for large arrays of vertices, normals and face indices. What is more, MongoDB provides full indexing, replica DBs as well as auto-sharding, Map/Reduce functionality and even geospatial indexing making it an attractive choice for production environments. Despite all of the aforementioned benefits, MongoDB is not a functional requirement of our framework and could be replaced by another suitable DB if desired.

By design, NoSQL databases pose no restrictions on the underlying layout of their collections (tables in relational DB terms). It is, therefore, possible to store any polymorphic documents in a single collection. Despite such a schema-less nature, it is important to enforce some basic rules about the DB structure. We assign two collections per 3D scene, one for all the scene graph constituents and one for all the documents that belong to a revision history. Hence, each of these collections stores a directed acyclic graph, making the access implementation reusable.

\subsubsection{DAG Representation}

To represent a directed acyclic graph in a flat collection of documents, we consider several common ways of graph storage:

Parental or child links Storing information about immediate parents or children of a node requires recursive hierarchical queries for graph retrieval. Unless there is a direct server-side DB support for it, such access would be computationally expensive.

Adjacency or incidence matrix Representing graph connectivity as a two-dimensional boolean matrix requires a complicated memory management when dealing with large graph structures.

Nested sets [Celko 2004] assigns each node two integers that represent boundaries of all of its children. In contrast to parental or child links, sub-tree retrieval can be implemented using a single query. However, inserting a new entry into DB causes boundary re-indexing on all the nodes. This was solved by using real numbers represented as quotients [Hazel 2008], although it is only suitable for trees where each node has exactly one parent.

Array of ancestors or materialized paths Storing in each node a full path from the root to itself makes retrieval of sub-graphs easy-all nodes with a given node in their path are its children. What is more, inserting additional nodes to such graph representation requires no modifications on the existing DB entries.

In a tree, there is always a single path to any node, however, in an arbitrarily complex directed graph such as $S G$ or $R H$, each possible path from the root would have to be stored, producing unnecessary 


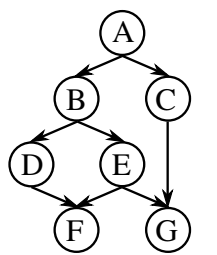

A: $[\mathrm{A}]$

$\mathrm{B}:[\mathrm{A} \triangleright \mathrm{B}]$

$\mathrm{C}:[\mathrm{A} \triangleright \mathrm{C}]$

$\mathrm{D}:[\mathrm{A} \triangleright \mathrm{B} \triangleright \mathrm{D}]$

$\mathrm{E}:[\mathrm{A} \triangleright \mathrm{B} \triangleright \mathrm{E}]$

$\mathrm{F}:[\mathrm{A} \triangleright \mathrm{B} \triangleright(\mathrm{D} \vee \mathrm{E}) \triangleright \mathrm{F}]$

$\mathrm{G}:[\mathrm{A} \triangleright((\mathrm{B} \triangleright \mathrm{E}) \vee \mathrm{C}) \triangleright \mathrm{G}]$

Figure 4: A simple DAG stored using the extended materialized paths notation. Each node lists all paths from root A to itself. Operator $\triangleright$ means parent-child relationship. Node $\mathrm{F}$, for example, can be reached either by path $[\mathrm{A} \triangleright \mathrm{B} \triangleright \mathrm{D} \triangleright \mathrm{F}]$ or (signified by $\vee$ ) by $[\mathrm{A}$ $\triangleright \mathrm{B} \triangleright \mathrm{E} \triangleright \mathrm{F}]$.

duplicates in the DB. We, therefore, extended the standard materialized paths to prevent data duplication. A logical disjunction $\vee$ is stored whenever multiple paths lead to the same node, see Fig. 4.

More formally, any such path is defined by a context-free grammar

$$
G=\left\{\{S, A\},\left\{n, n_{\text {root }}, \epsilon\right\}, P, S\right\},
$$

where $\epsilon$ is an empty string, $n \in S G-\left\{n_{\text {root }}\right\}$ and $P$ is the set of rules

$$
\begin{aligned}
& S \rightarrow\left[n_{\text {root }} \triangleright A\right], \\
& A \rightarrow \epsilon|n|(A)|A \triangleright A|(A \vee A) .
\end{aligned}
$$

Here, $\triangleright$ operator signifies a parent-child relationship on a scene graph while $\vee$ a logical disjunction if there is more than one possible path from $n_{\text {root }}$ to $n$.

\subsubsection{Scene Graph as BSON}

BSON is designed to be efficient for scanning and parsing, however, in some cases, it can occupy more space than JSON equivalent, especially as it explicitly encodes array indices. Therefore, our implementation stores large vertex and normal arrays as binary entries within BSON documents to avoid unnecessary data overhead. At the moment, such byte arrays are assumed to be in 32-bit littleendian ordering. Hence, it is easy to perform an early reject byteby-byte memory comparison on these fields to determine whether they are identical to those already stored in the repository. If, for example, the number of vertices on a mesh document differs from that in the head revision, it is flagged as modified. Nevertheless, even a full binary comparison is a reasonably fast operation. Given the linear approach, our solution scales well to very large model sizes. The ID part of the revision metadata is recorded as a universally unique identifier (uuid), as defined by ITU-T [2008]. This is especially suitable as it enables committing changes to a single DB without ID clashes in a distributed manner. In addition, our implementation supports shader programs to be part of a scene graph. We store the vertex and fragment shader definitions together as strings respectively. These can be compiled by the viewer application.

\subsection{D Revision Control Viewer}

Our example of a tier 1 application for repository interaction and asynchronous revision control, 3D RCViewer, is written in $\mathrm{C}++$ and a cross-platform UI framework Qt [Blanchette and Summerfield 2008]. Making use of the Open Asset Import Library (Assimp) [Schulze et al. 2012], it converts common 3D file formats such as Collada, Blender 3D, 3ds Max, Wavefront Object etc. into a unified in-memory scene graph representation ${ }^{2}$. This is subse-

\footnotetext{
${ }^{2}$ Assimp currently exports into Collada, Wavefront Object and Stereolithography formats with many more in development.
}

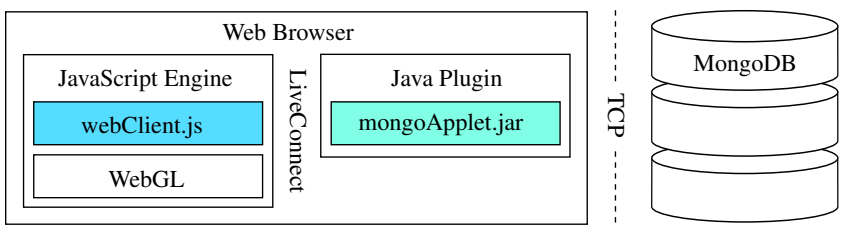

Figure 5: 3D Web Client implementation. Client-side JavaScript leverages the MongoDB Java driver (exposed via a custom applet) to query BSON documents from a $3 D$ Repository. JavaScript-toJava communication is possible thanks to the LiveConnect feature of the Java browser plugin. Once the scene graph is reconstructed, WebGL renders the retrieved geometry inside a web browser.

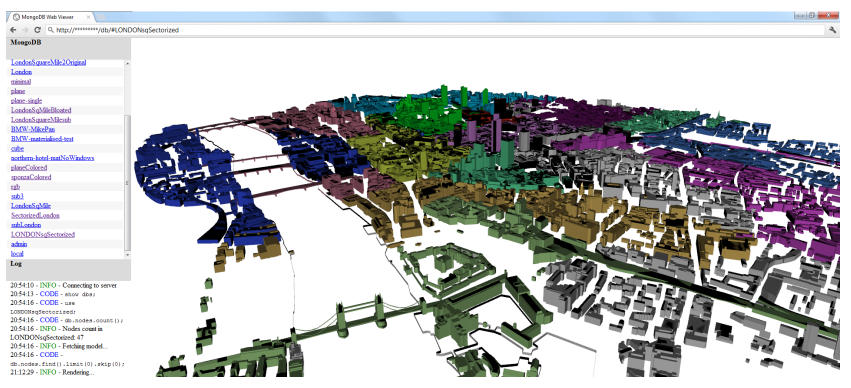

Figure 6: Part of a sectorized $3 D$ model of London displayed in Chrome web browser. Original scene was exported from Rhinoceros $3 D$ as a $3 d s$ Max file (*.3ds). Over $340 k$ polygons are loaded as Blender (*.blend) and Wavefront (*.obj) files in different revisions. Model courtesy of Arup.

quently converted into a flat list of BSON objects that can be stored in MongoDB (acting as a 3D repository). The UI supports standard delta commits, revision retrieval and 3D diff as shown in Fig. 3. A color-coded list further enables the user to quickly examine the differences and choose one of the two conflicted revisions for each $S G$ node. Alternatively, if a more complicated merge is required, it is possible to export conflicted nodes into a separate 3D file for additional editing.

\subsection{D Web Client}

3D Web Client is an example of a tier 2 read-only DB front-end application. Written as a combination of MongoDB Java driver and JavaScript WebGL renderer, this client-side implementation displays 3D models from the repository via common web browsers. Plenty of work has been done on the server-side management of MongoDB via JavaScript (JS), however, there currently exists no client that would communicate with the DB via BSON objects directly. Once the HTML5 WebSocket API proposal [W3C 2012] gains full support across popular web browsers, it would be feasible to implement a dedicated browser-based client via JS and the WebSocket protocol. Until then, the most appropriate approach is to rely on the native MongoDB Java driver which takes care of the DB connections and data delivery. Even when the WebSocket proposal is approved, a dedicated client interface will still be needed.

\subsubsection{LiveConnect}

Formerly Mozilla-specific, the new LiveConnect Java plugin feature enables the communication between the Java Virtual Machine (JVM) and the JavaScript Engine running in a web browser [Oracle Corporation 2012]. On one hand, a Java applet can invoke scripts on a page and populate JS objects. On the other hand, JavaScript 


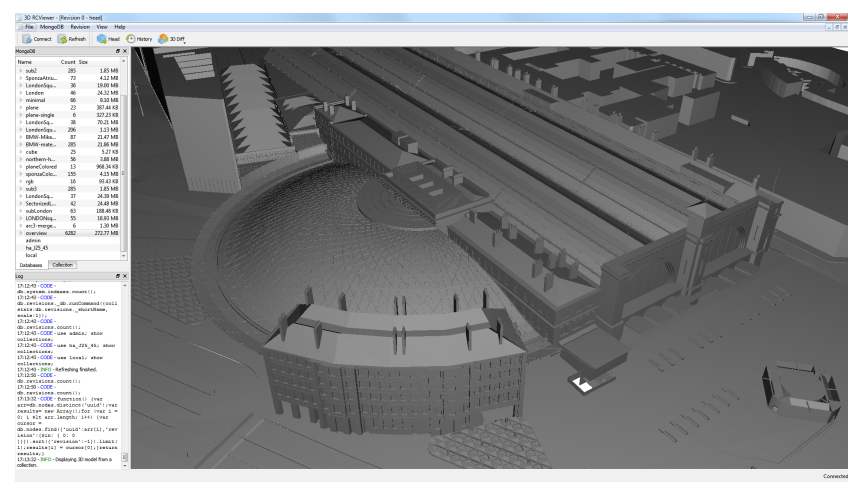

Figure 7: Model of the King's Cross station redevelopment loaded from our repository. The building in Fig. 1 is a subset of this 2.6M polygon scene. Model courtesy of Network Rail.

can access Java runtime libraries, static methods, create objects and execute public methods on Java applets.

To make our implementation extensible to the WebSocket integration in the near future, we rely on the default MongoDB Java driver version 2.7.3 as a library only. We create an applet wrapper which exposes useful methods to the JavaScript engine, but has no execution logic to manage the WebGL client itself. In addition, we include static methods to convert between the BSON document collection retrieved from a MongoDB repository and a scene graph representation required by WebGL. Once the scene graph is retrieved and reconstructed, our JavaScript webClient application renders the entire model using WebGL, see Fig. 6. Implementation overview is depicted in Fig. 5.

\section{Examples}

In this section, we demonstrate some of the main features supported by our framework. Additional examples are provided as supplementary materials.

1. As shown in Fig. 1, it is possible to retrieve any revision from the repository and visualize it within a web browser. Here, examples of both tiers of applications are present. The first is tier 1 with read-write privileges while the second is a read-only viewer.

2. Fig. 3 is a typical example of our $3 \mathrm{D}$ conflict resolution interface (3D Diff tool). Two versions of the same model, one from the repository and one as a local file, are loaded into the 3D RCViewer GUI to be compared against their common ancestral model, also loaded from the repository (head revision).It detects conflicts (in red) and in a 3-way manner identifies nonconflicting edits (in blue) that can be automatically merged.

3. Visualized in the 3D RCViewer in Fig. 7 is an example of a large scene with several million polygons being handled by our framework. The building model in Fig. 1 is a subset of this $3 \mathrm{D}$ scene that can be retrieved and tracked independently, see $\S 4$.

4. Finally, Fig. 8 demonstrates the actual changes stored within the repository. Those scene graph components that have not been modified are queried from their latest revision, hence the requirements for storage are relatively small in comparison to other tracking methodologies.

\section{Discussion}

The interaction between the modeling software and our 3D Repository takes place through the import and export of files via $3 \mathrm{D}$
RCViewer. We have succeeded in decoupling the modeling from the long-term storage, as the files are now considered to be only a temporary representation to facilitate data interchange with a variety of editors. We consider this to be of great importance as the range of editing software and other mesh generation tools is vast.

\subsection{Limitations}

By definition, the smallest unit of change in our prototype is a BSON document, where arrays are used for vertex coordinates, indices etc. This is efficient if the likely access is a collection of documents, but is not if edits are very localized. If, for example, a single vertex was repositioned in a mesh object, only its document would replace the previous version in the next revision, not the whole scene. This level of granularity might not be suitable for all projects, however, it would certainly be possible to support multiple types of object representations within the same DB. The repository interface could store small edits as operations depending on the structure of the DB collections (tables).

When interacting with our revision control framework, the file representations need to convey changes efficiently. That is, the system must be able to detect that the imported nodes match the existing entries in the repository and that some of them have changed since the previous revision. At the moment, we rely on the assumption that imported 3D assets preserve revision metadata (ID and a revision number) for each component. However, there is no guarantee that the editing software will do this and users will always be able to modify the data if they wish. Thus, an interesting matching problem occurs. It is possible to compare SG nodes against all the previous versions stored in the DB. This is speeded up by relying on the data sizes as an early indicator of correspondence and the fact that matching is a pure binary comparison. To go further, it would be necessary to support the matching of arbitrary meshes against each other, an interesting research problem in itself (easily circumvented by a direct editor connection to the repository, see $\S 7.2$ ).

A serious problem with schema-less NoSQL databases like MongoDB is the lack of data validation on insertion, unless specified as a unique key. Hence, it would be necessary to implement a serverside deamon acting as a DB interface for a production-ready implementation of our framework. This would need to facilitate revision control, analyze commits and check for consistencies instead of relying on a GUI implementation.

Web Client Our WebGL-based client is limited by the underlying OpenGL ES 2.0 specification, such as the ability to render only a limited number of indexed vertices $\left(2^{16}\right.$, same as X3D). Furthermore, MongoDB currently offers an increased upper limit of 16 MB per BSON document. Hence, there is a potential need to split larger meshes and textures into smaller sections to meet such criteria. This, however, should occur only rarely as many file formats have lower size limits. In addition, LiveConnect treats JavaScript as if originating from an untrusted source restricting its ability to open any crossdomain Java connections, see the security model reference in [Oracle Corporation 2012]. Therefore, our web client has to be loaded from the same server on which the MongoDB is hosted, a small yet important limitation.

\subsection{Other Interfaces}

As suggested in Fig. 2, the GUI applications are not a necessary component to build the 3D Repository. The two applications that connect to the DB and facilitate revision control are mainly prototype examples that demonstrate the feasibility of our solution and this family of applications could easily be extended. Given the Java 


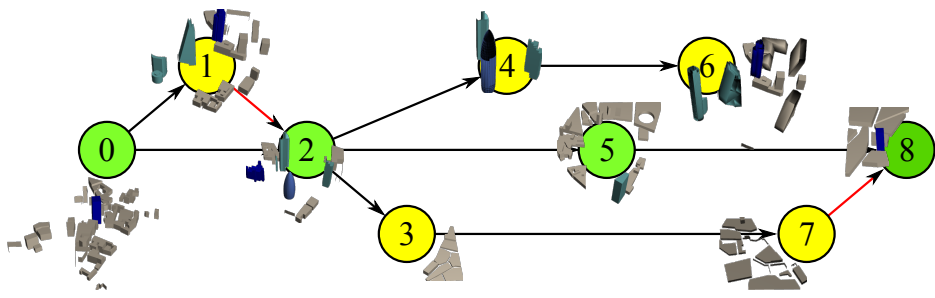

(a) Revision history

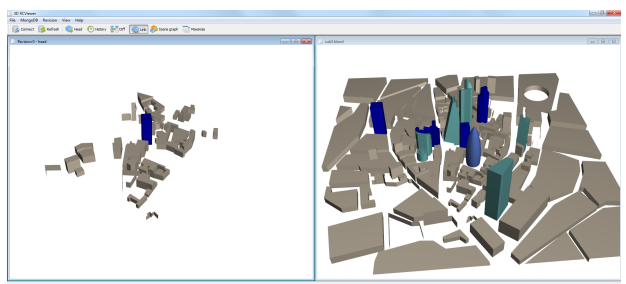

(b) Revisions 0 and 8 respectively

Figure 8: (a) A schematic representation of a revision history shows delta changes evolving over several branches. The trunk is shown in green, branches in yellow and merges in red. (b) the initial revision 0 (right) versus the final revision 8 (left).

driver we employed and the support for OpenGL ES rendering, an Android-specific tier 2 viewer has been developed. The editing software could itself replace the tier 1 GUI and connect to the DB directly (dashed arrow). The plug-in frameworks provided by many modern editors such as Autodesk 3ds Max or Blender could connect to the DB directly, exploiting all the data available in a repository. In particular, on committing changes back to the DB, the system would know exactly at which point an asset was retrieved and which revision it belongs to. Thus, the internal workings of the revision control could be entirely hidden from the user. It is important to note that unless some form of access control is in place (see $\S 7.3$ ), conflict resolution as defined by our 3D Diff is still necessary.

\subsection{Feature Extensions}

An obvious feature that could be added is the access control via locking. Even though it is not difficult to add to the DB schema, it is difficult to preserve such locks in the round-trip to the file store. What is more, it is not trivial to decide the granularity level the locks should be on. One can imagine supporting read/write locks that would prevent conflicts arising altogether. Hybrid types of locks, on the other hand, could enforce geometric constraints on the model. A single polyline in such a case could define a locked interface between a piece of geometry to be modified and the rest of the model. Addressing this is planned for the future work.

Another feature to add would be the facility to search for assets via spatial queries, reminiscent of the role of spatial DBs. To achieve this, a bounding box hierarchy could be provided. This could be used both for general queries ("fetch all objects within this region", "fetch all objects adjoining given objects"), but also to facilitate detection of matches when the revision metadata has been lost. MongoDB offers native geospatial indexing to further support such a repository extension. Based on this, we plan to devise a BSONbased 3D protocol for real-time online visualization.

\section{Conclusions}

We have presented a novel approach to storage and asynchronous revision control of 3D assets via a NoSQL DB. Our custom-made database front-ends feature version tracking, differencing and conflict resolution as well as remote repository visualizations. We believe that the unification of storage and versioning in a single DB can provide significant benefits over the common practice today which uses standard version control systems and asset tracking.

We have successfully decoupled 3D modeling from its long-term storage by creating a suitable repository in a standard release MongoDB. Firstly, we addressed the issues of representing hierarchical scene graphs in such a DB and selected the size and types of elements stored. On top of this, we created a revision control system that allows for branching and merging, enabling concurrent editing on the repository. In creating our framework, we decided to support 3D editing using the broadest range of tools; thus relying on import and export of files in the standard formats rather than on a specific modeling tool. In addition, we captured the scene specific information alongside of $3 \mathrm{D}$ models within the same repository.

There are many avenues for future work. Some of these are discussed in $\S 7$ in the context of limitations and potential benefits of our approach. Our intention is to build this into a robust tool that can be used by the community, thus making this work open source in the near future. Our framework will hopefully facilitate crowdsourcing of models for various purposes. In the next release, we will expand our implementation to capture textures in the DB, include an Android-specific viewer, promote our 3D Web Client into a tier 1 application that can import and export 3D models and implement a plug-in based access for one or more standard 3D modeling packages with locking mechanism enabled.

\section{Acknowledgements}

This research was kindly sponsored by Arup Foresight and the UK EPSRC-funded Eng. Doctorate Centre in Virtual Environments, Imaging and Visualisation (EP/G037159/1). We would like to thank Kenny Mitchell at the former Black Rock Studio and Alvise Simondetti at Arup for the discussions that informed our work. Part of the inspiration for this work came when the second author was on a sabbatical at Electronic Arts (EA). Finally we would like to thank the reviewers for their valuable comments.

\section{References}

10GEN, INC., 2010. BSON-binary json specification, Feb. http: //bsonspec.org/.

Austerberry, D. 2006. Digital asset management. Focal.

BARnes, M., AND Finch, E. L. 2008. Collada - digital asset schema release 1.5.0. Specification, Khronos Group, Apr.

Behr, J., Jung, Y., Keil, J., Drevensek, T., Zoellner, M., ESCHLER, P., AND FELLNER, D. 2010. A scalable architecture for the $\mathrm{html} 5 / \mathrm{x} 3 \mathrm{~d}$ integration model x3dom. In Proceedings of the 15th International Conference on Web 3D Technology, ACM, New York, NY, USA, Web3D '10, 185-194.

Bentley Systems, 2011. Assetwise, Sep. http://www. bentley.com/en-GB/Products/assetwise/.

Berthelot, R. B., Royan, J., Duval, T., and Arnaldi, B. 2011. Scene graph adapter: an efficient architecture to improve interoperability between $3 \mathrm{~d}$ formats and $3 \mathrm{~d}$ applications engines. 
In Proceedings of the 16th International Conference on $3 D$ Web Technology, ACM, New York, NY, USA, Web3D '11, 21-29.

Blanchette, J., And Summerfield, M. 2008. $C++G U I$ Programming with Qt4, second ed. Prentice Hall, Feb.

BRINK, E., AND SteenberG, E., 2011. Uni-verse main page, Sep. http://www. uni-verse.org.

Celko, J. 2004. Joe Celko's Trees and Hierarchies in SQL for Smarties. Morgan Kaufmann, May.

Chen, H.-T., WeI, L.-Y., AND Chang, C.-F. 2011. Nonlinear revision control for images. ACM Trans. Graph. 30, 4 (Aug), 105:1-105:10.

Connolly, T., And BegG, C. 2004. Database Systems: A Practical Approach to Design, Implementation and Management. Addison Wesley. ISBN: 978-0321210258.

DAsSault Systemes, 2011. Enovia, September. http:// www.3ds.com/products/enovia/solutions/.

DeBiswas, K., AND RaO, N., 2012. Sunglass, January. http: //sunglass.io.

Denning, J. D., KerR, W. B., ANd Pellacini, F. 2011. Meshflow: interactive visualization of mesh construction sequences. ACM Trans. Graph. 30, 4 (Aug), 66:1-66:8.

Doboš, J., AND STEEd, A. 2012. Revision Control Framework for 3D Assets. Eurographics Association, Cagliari, Sardinia, Italy, A. Fusiello and M. Wimmer, Eds., 1-2.

DouRISH, P. 1995. The parting of the ways: divergence, data management and collaborative work. In Proceedings of the fourth conference on European Conference on Computer-Supported Cooperative Work, Kluwer Academic Publishers, Norwell, MA, USA, 215-230.

HAZEL, D. 2008. Using rational numbers to key nested sets. CoRR abs/0806.3115.

Hesina, G., Schmalstieg, D., Furhmann, A., And PurGATHOFER, W. 1999. Distributed open inventor: a practical approach to distributed 3d graphics. In Proceedings of the ACM symposium on Virtual reality software and technology, ACM, New York, NY, USA, VRST' 99, 74-81.

Hunt, J. J., Vo, K.-P., And Tichy, W. F. 1998. Delta algorithms: an empirical analysis. ACM Trans. Softw. Eng. Methodol. 7 (Apr), 192-214.

Jacobsen, J., Schlenker, T., And Edwards, L. 2005. Implementing a digital asset management system: for animation, computer games, and web development. Elsevier Focal Press.

JAIN, A., ThormäHLEN, T., Ritschel, T., AND SEIDEL, H.-P. 2012. Exploring shape variations by 3d-model decomposition and part-based recombination. Comp. Graph. Forum (Proc. Eurographics 2012) 31, 2.

Kay, L., Osowski, A., Lindeque, R., Brys, S., AND BANNASCH, S., 2009. Scenejs. http: // scenejs .org.

Koller, D., Frischer, B., And Humphreys, G. 2010. Research challenges for digital archives of $3 \mathrm{~d}$ cultural heritage models. J. Comput. Cult. Herit. 2, 3 (Jan), 7:1-7:17.

Malewicz, G., Austern, M. H., Bik, A. J., Dehnert, J. C., Horn, I., Leiser, N., AND CzAJKowski, G. 2009. Pregel: a system for large-scale graph processing. In Proceedings of the 28th ACM symposium on Principles of distributed computing, ACM, New York, NY, USA, 6-6.
Membrey, P., Plugge, E., And Hawkins, T. 2010. The Definitive Guide to MongoDB: The NoSQL Database for Cloud \& Desktop Computing, first ed. APRESS ACADEMIC.

MENS, T. 2002. A state-of-the-art survey on software merging. IEEE Transactions on Software Engineering 28, 5, 449-462.

Obe, R., And Hsu, L. 2011. PostGIS in Action, first ed. Manning Publications.

ORACLE CORPORATION. 2012. Liveconnect support in the new Java $^{\mathrm{TM}}$ plug-in technology. Tech. rep., Java SE 6 update 10. http://jdk6.java.net/plugin2/liveconnect.

Pilato, C. M., Collins-Sussman, B., AND W. FitzPatrick, B. 2008. Version Control with Subversion, second ed. O'Reilly Media, Sep.

RigauX, P., Scholl, M., And Voisard, A. 2001. Spatial Databases: With Application to GIS. Morgan Kaufmann. ISBN: 978-1558605886.

Schulze, T., Gessler, A., Kulling, K., NAdlinger, D., Klein, J., Sibly, M., AND Gubisch, M., 2012. Open asset import library (assimp), Jan. http://assimp. sourceforge.net/.

SinK, E. 2011. Version Control by Example, first ed. Pyrenean Gold Press.

Sons, K., Klein, F., Rubinstein, D., Byelozyorov, S., And SLUSALLEK, P. 2010. Xml3d: interactive 3d graphics for the web. In Proceedings of the 15th International Conference on Web 3D Technology, ACM, New York, NY, USA, Web3D '10, 175-184.

Steed, A., AND Oliveira, M. 2009. Networked Graphics: Building Networked Games and Virtual Environments. Elsevier. ISBN-10: 0123744237.

Telecommunication Standardization Sector. 2008. Generation and registration of universally unique identifiers (uuids). Rec, ITU, Aug. X.667, ISO/IEC 9834-8.

Unity TECHNOLOGIES, 2010. Using external version control systems with unity.

http://unity3d.com/support/documentation/ Manual/ExternalVersionControlsystemSupport. html/.

V. Kothuri, R., Godfrind, A., And Beinat, E. 2012. Pro Oracle Spatial for Oracle Database 11g. Apress Academic, Jan.

VISTRAILS, INC., 2012. Vistrails for maya, Jan. http: //www . vistrails.com/maya.html.

W3C, 2012. The websocket api - editor's draft, March. http: //dev.w3.org/html5/websockets/.

W ÄSCH, J., AND KLAS, W. 1996. History merging as a mechanism for concurrency control in cooperative environments. In RIDE '96 Interoperability of Nontraditional Database Systems, IEEE Computer Society, 76-.

Web3D Consortium, 2008. Extensible 3d (X3D). ISO/IEC 19775:200x, http://www.web3d.org/x3d/ specifications/x3dspecification.html.

Wingerd, L. 2005. Practical Perforce, first ed. O'Reilly Media.

Zeleznik, B., Holden, L., Capps, M., Abrams, H., AND Miller, T. 2000. Scene-graph-as-bus: Collaboration between heterogeneous stand-alone 3-d graphical applications. In Eurographics. 